\title{
Überlebensvorteil durch Erhaltungstherapie mit Rituximab
} Nach einer erfolgreichen Induktionsbehandlung kann eine Erhaltungstherapie mit Rituximab das progressionsfreie Überleben von
Patienten mit follikulärem Lymphom verlängern. Das belegen die aktuellen Ergebnisse der PRIMA-Studie.

In der Studie PRIMA (Primary Rituximab and Maintenance) erreichten 1.018 Patienten mit fortgeschrittenem follikulärem Lymphom nach einer Induktion mit Rituximab und Chemotherapie eine komplette oder partielle Remission (Salles GA et al., 2010, ASCO: \#8004). Danach wurden sie randomisiert mit Rituximab (MabThera ${ }^{\oplus}, 375 \mathrm{mg} / \mathrm{m}^{2}$ KOF alle 8 Wochen) für 2 Jahre $(n=505)$ weiterbehandelt oder beobachtet $(n=513)$. Die Interimsanalyse nach 25 Monaten ergab einen signifikanten Vorteil beim progressionsfreien Überleben (PFS) zugunsten der Erhaltungstherapie (82\% versus 66\%; Hazard-Ratio 0,50; $p<0,0001)$. Der Vorteil beim
PFS war unabhängig von Alter, Geschlecht, Risikostratifikation und eingesetztem Chemotherapieprotokoll in der Induktionstherapie, so Michael Herold, Erfurt. Aufgrund dieser Daten wurde inzwischen von der Europäischen Kommission die Zulassung für die Rituximab-Erhaltungstherapie in der First-line-Therapie des follikulären Lymphoms erteilt.

\section{Positive Erfahrungen in der Praxis}

Mathias Witzens-Harig, Heidelberg, präsentierte den Fall eines 65-jährigen Patienten mit follikulärem Lymphom (WHO-Grad II) und einem FLIPI (Follicular Lymphoma In- ternational Prognostic Index) von 4. Nach 2-jähriger Beobachtung kam es bei dem Patienten zur Progression. In der Biopsie wurde jetzt ein follikuläres Lymphom vom WHO-Grad IIIA festgestellt. Unter einer Therapie mit 6 Zyklen Rituximab-CHOP (Cyclophosphamid/Doxorubicin/Vincristin/Prednison) 21 und einer anschließenden Monotherapie mit 2 Zyklen Rituximab erreichte der Patient eine partielle Remission. Im Anschluss erhielt er eine Erhaltungstherapie mit Rituximab für 2 Jahre $\left(375 \mathrm{mg} / \mathrm{m}^{2}\right.$ KOF alle 2 Monate) und ist seitdem in kompletter Remission. $A A A$

Quelle: Veranstaltung der Roche Pharma AG

\section{Myelodysplastische Syndrome}

\section{Einfluss der Eisenchelationstherapie auf das Überleben}

\begin{abstract}
Effektive risikoadaptierte Therapiestrategien haben die Prognose der Patienten mit myelodysplastischen Syndromen (MDS) in den letzten Jahren deutlich verbessert. Entscheidend ist in mehr als $90 \%$ der Fälle eine gute supportive Therapie, bei der die Substitution mit Erythrozytenkonzentraten im Vordergrund steht.
\end{abstract}

Eine hohe Transfusionslast, die mit steigenden Serumferritinwerten einhergeht, ist für MDS-Patienten allerdings ein negativer prognostischer Faktor für die allogene Stammzelltransplantation. Experten fordern daher eine konsequente Eisenchelattherapie für MDS-Patienten mit Eisenüberladung, um die Lebenserwartung zu verlängern. Empfohlen werden kann eine Eisenchelattherapie bei Patienten mit einem Serumferrintinwert von $>1.000 \mathrm{ng} / \mathrm{ml}$, die für mindestens $1 \mathrm{Jahr}$ mehr als 2 Erythrozytenkonzentrate monatlich erhalten, auf eine primäre Therapie nicht ansprechen oder für eine Stammzelltransplantation vorgesehen sind, sowie bei Patienten mit beeinträchtigter Organfunktion (Bennett JM et al., 2008, Am J Hematol 83: 858-861).

\section{Studien belegen Vorteile der Eisenchelationstherapie}

Aktuelle Daten aus einer prospektiven nicht randomisierten Studie zeigen, dass MDS-Patienten, die nach IPSS (International Prognostic Scoring System) der Risikogruppe "Niedrigrisiko" oder "Intermediär-1“ angehören, unter regelmäßiger Anwendung von Eisenchelatoren einen signifikanten Überlebensvorteil ha-

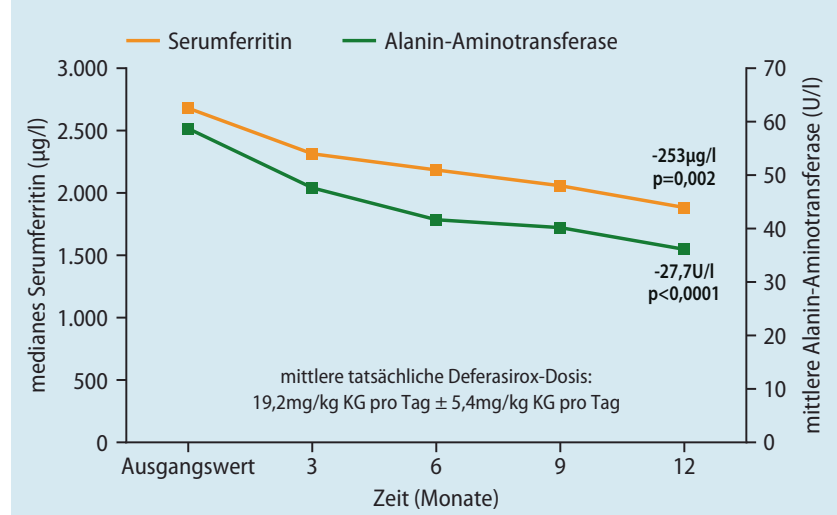

nach Gattermann N et al., 2010, Leuk Res 34: 1143-1150

EPIC-Studie - Reduktion der Alanin-Aminotransferase-Serumwerte und des Serumferritins unter Deferasirox

ben (median 138 Monate versus 70 Monate bzw. 115 Monate versus 36 Monate jeweils ohne Eisenchelattherapie; $p=0,015$ bzw. $p=0,003$; Rose C et al., 2010, Leuk Res 34: 864-870).

In der Studie EPIC (Evaluation of Patients' Iron Chelation with Exjade $^{\circledast}$ ) zur Eisenchelationstherapie mit Deferasirox zeigte sich außerdem eine Verbesserung der Leberfunktion (Abb.). UF Quelle: Veranstaltung der Novartis Pharma GmbH 